\title{
The ComQXPA Quorum Sensing System May Play an Important Role in the Synthesis of Bacillomycin D in Bacillus Amyloliquefaciens Q-426
}

chunshan quan ( $\square$ mikyeken@dlnu.edu.cn )

Dalian Minzu University

liming jin

Dalian Minzu University

wei zhou

Dalian Minzu University

jialu liu

Dalian Minzu University

xian shi

Dalian Minzu University

wei zheng

Dalian Minzu University

jing liu

Dalian Minzu University

ruochen fan

Dalian University of Technology

liying zhang

Dalian Minzu University

pengchao zhao

Henan University of Science and Technology

shengdi fan

Dalian Minzu University

\section{Research Article}

Keywords: Bacillus amyloliquefaciens Q-426, ComQXPA, Quorum sensing, ComXQ-426, Lipopeptides

Posted Date: December 30th, 2020

DOI: https://doi.org/10.21203/rs.3.rs-129057/v1 
License: (c) (i) This work is licensed under a Creative Commons Attribution 4.0 International License. Read Full License 


\section{Abstract}

Background: Bacillus amyloliquefaciens Q-426 can secrete numerous cyclic lipopeptides that have antifungal and antitumor activities. ComQXPA is a common quorum sensing (QS) system in Bacillus species. Most $B$. amyloliquefaciens strains are encoding the QS gene cluster comQXPA, however, the biological function of the ComQXPA system in $B$. amyloliquefaciens has not been well studied. In this study, we identified the comQXPA gene locus and the chemical structure of $\mathrm{ComX}_{\mathrm{Q}-426}$ in $B$. amyloliquefaciens Q-426, and explored the function of $\mathrm{ComX}_{\mathrm{Q}-426}$ in regulating lipopeptide production.

Results: We identified and analyzed the comQXPA locus in Q-426. The full length of the comQXPA gene cluster was 4,014 bp, including $912 \mathrm{bp}$ of comQ, $165 \mathrm{bp}$ of $\operatorname{comX}, 2292 \mathrm{bp}$ of comP, and $645 \mathrm{bp}$ of comA. The comQXPA locus belongs to group $\mathrm{B}$, as $\operatorname{com} Q$ and $\operatorname{com} X$ overlap by only one base pair. $\operatorname{ComX}_{\mathrm{Q}-426}$ consists of six amino acids (GGDWKY) that contain a modified tryptophan residue. The antifungal activity of $Q 426 \triangle$ comX was significantly affected, and almost no antifungal activity was observed, while the antifungal activity of strain Q426 $\triangle \operatorname{com} X / \operatorname{com} Q X$ was restored to the same level as that of the wildtype strain. When the ComX-426 was added to the culture medium at a final concentration of $8 \mu \mathrm{g} / \mathrm{L}$ at the early stage of the log-phase, the antifungal activity of the wild-type strain Q-426 was significantly improved. Knocking out the comX gene did not affect the growth of the bacteria, however, the strain Q426AcomX lost its swimming ability, was unable to form colonies when spread on a solid surface, and could not form biofilms on the interface between the gas and liquid medium.

Conclusions: Disruption of the ComPA signaling pathway in the Q-426 strain resulted in significant effects on bacillomycin D production, morphology, and motility.

\section{Background}

Bacillus amyloliquefaciens, a Gram-positive and soil-dwelling bacterium, is a species in the genus Bacillus with high similarity to Bacillus subtilis. During growth, $B$. amyloliquefaciens can produce a wide range of secondary metabolites (surfactins, iturins, and fengycins) that have antagonistic effects on fungal pathogens such as Fusarium graminearum and Rhizopus stolonifera [1-3]. Therefore, $B$. amyloliquefaciens is considered a good candidate for biocontrol [4-6].

Bacteria coordinate their group behavior through quorum sensing (QS), whereby cells secrete diffusible signal molecules called autoinducers that activate a cognate receptor to control a wide array of quorum sensing responses in a density-dependent manner. In most Gram-positive bacteria, quorum responses are controlled by signaling peptides that are typically produced from oligopeptide precursors. In B. subtilis, quorum responses contribute to antibiotics production, the induction of competence development, sporulation, and a multitude of social (multi-cellular) behaviors, including swarming and sliding motility, exoprotease production, and biofilm formation [7-10]. 
The ComQXPA QS system of $B$. subtilis is a typical QS system of Bacillus bacteria; the system controls the expression of nearly 200 genes, including both extracellular and intracellular factors [11]. This QS system consist of an isoprenyl transferase (ComQ), an autoinducer (ComX), a histidine kinase (ComP), and a response regulator (ComA). The autoinducer $\operatorname{ComX}$ is initially synthesized as a 47-73 residue propeptide (pre-ComX) and then processed and modified by ComQ. Extracellular accumulation of the modified ComX leads to phosphorylation of ComA, and then the phosphorylated ComA directly modulates the expression of various genes, including the sifA operon required for surfactin biosynthesis [12-16].

Several studies have indicated that the comQXPA gene cluster is widespread in Bacillus and that it exhibits a remarkable degree of intraspecific diversity $[14,15,17,18]$. These studies have shown that the locus encoding the ComQXPA system is highly polymorphic, especially the coding regions for comQ, $\operatorname{com} X$, and the $5^{\prime}$-end of comP, which are poorly conserved. However, the conservation of the genes followed the same order: Com $A>\operatorname{ComP}>\operatorname{ComQ}>\operatorname{ComX}$. The genomes of many bacteria contain substantial fractions of overlapping co-directional gene pairs (resulting in overlapping reading frames), and gene overlaps are known to play an important role in their evolution and biological function $[19,20]$. Dogsa et al. performed comparative experiments with comQXPA-like gene arrangements in 2620 complete and 6970 draft prokaryotic genomes. Their results demonstrated that in addition to $B$. subtilis and close relatives, other bacteria species have comQXPA-like loci and characteristic gene-overlap patterns as in the comQXPA loci. Dogsa and co-workers divided the types of gene-overlaps into five groups based on the number of overlaps in the reading frames and the number of amino acids (Fig. S1). Group A exhibits no overlapping reading frames between genes of $\operatorname{com} Q, \operatorname{com} X, \operatorname{com} A$, and $\operatorname{com} P$, group $B$ exhibits an overlapping reading frame between $\operatorname{com} Q$ and $\operatorname{com} X$; group $C$ exhibits a continuous overlapping reading frame between $\operatorname{com} Q, \operatorname{com} X$, and $\operatorname{com} P$; group $\mathrm{D}$ exhibits an overlapping reading frame between comP and $\operatorname{com} A$, and group $E$ exhibits a continuous overlapping reading frame between com $X, c o m P$, and comA. The overlap types are dominated by an apparent mutation in the com $Q$ stop codon that results in a 13-18 amino acid long C-terminal extension [19].

The ComX pheromone is a post-translationally modified oligopeptide. The modification is catalyzed by ComQ and is essential for the function of the pheromone. Previous studies have demonstrated that striking polymorphism occurs in the amino acid sequence of the ComX pheromone in certain Bacillus species, but each ComX pheromone possesses a tryptophan residue at either the 3rd or 4th residue from the $\mathrm{C}$-terminus. Okada et al. noted that the tryptophan residue is modified with either a geranyl group or a farnesyl group at its $y$-position, resulting in the formation of a tricyclic structure that includes a newlyformed proline-like five-membered ring $[17,21-28]$.Thus far, the chemical structures of only seven ComX pheromones have been identified.

In previous studies, we reported that $B$. amyloliquefaciens $Q-426$ isolated from compost exhibits strong antifungal activity and produces various lipopeptides, including bacilomycin $D$, fengycin $A$, and fengycin $B$ [29-31]. Whether the production of those lipopeptides is directly regulated by ComQXPA QS system and is a critical function for cell survival of $B$. amyloliquefaciens Q-426 remains unclear. Here, we 
identified and analyzed the comQXPA locus of $B$. amyloliquefaciens Q-426. Moreover, we identified the chemical structure of $\mathrm{ComX}_{\mathrm{Q}-426}$ and investigated its function in regulating lipopeptide production and its biological characteristics. We found that the production of lipopeptide-like antifungal compounds is controlled by the ComPA signaling pathway in $B$. amyloliquefaciens Q-426, and we demonstrate that a lack of production of lipopeptides affects the swarming ability and biofilm formation of Q-426.

\section{Results}

\section{Gene overlapping in the comQXPA locus of B. amyloliquefaciens Q-426}

The entire comQXPA gene cluster was amplified from the chromosomal DNA of $B$. amyloliquefaciens Q426. The full length of the comQXPA gene cluster was 4,014 bp, including $912 \mathrm{bp}$ of comQ, $165 \mathrm{bp}$ of comX, $2292 \mathrm{bp}$ of comP, and $645 \mathrm{bp}$ of comA. The chromosomal arrangement of the comQXPA locus in strain Q-426 is shown schematically in Fig. $1 \mathrm{~A}$.

In Bacillus, each gene in the comQXPA locus is differentially conserved and overlapped. Approximately half of the comQXPA loci exhibit no overlapping gene pairs belonging to group $A ; 31.6 \%$ of the loci contain overlapping gene pairs belonging to group $B$ and only a few loci belonging to groups $C, D$ and $E$ [19]. The comQXPA locus in strain Q-426 belongs to group B, because only one base pair overlapped between $\operatorname{com} Q$ and $\operatorname{com} X$.

We analyzed 40 comQXPA sequences from completely annotated Bacillus genomes that had at least one lipopeptide synthesis gene cluster, for example iturins, surfactins, and fengycins. Some of the sequences were from important commercial strains ( $B$. velezensis QST713, B. velezensis FZB42). A phylogenetic tree was constructed based on the comQ gene sequences, as the comQ gene shows high diversity in the comQXPA locus. The gene overlapping types are marked in different colors in the Fig. 2 . As shown in Fig. 2, most of the comQXPA loci belong to group $B$, and the number of overlapping base pairs varied from 1 to 49 base pair overlaps. There was no corresponding relationship between species and overlapping type, and the relationship between the number of overlaps and the function was unclear.

Many of $B$. amyloliquefaciens strains were registered as biological control agents and are commercially available because they can produce numerous antifungal metabolites with well-established activity in vitro such as bacillomycin, fengycin, and surfactin $[32,33]$. $B$. amyloliquefaciens is one member of the $B$. subtilis group that includes $B$. subtilis, $B$. licheniformis, $B$. pumilus, and $B$. amyloliquefaciens. However, these species were frequently misidentified for many years due to the lack of distinguishing phenotypes, and they are poorly resolved by $16 \mathrm{~S}$ rRNA sequencing. In recent years, the taxonomy of the $B$. subtilis species group has been updated and clarified based on their whole genome sequences. Some of the strains that were previously registered as $B$. subtilis or $B$. amyloliquefaciens are actually strains of $B$. velezensis [34, 35]. For example, some important commercial strains, $B$. subtilis QST713 and $B$. amyloliquefaciens subsp. plantarum FZB42, were reidentified as $B$. velezensis QST713 and $B$. velezensis FZB42 based on phylogenetic analysis [36,37]. We also found that many strains belonging to the $B$. 
subtilis group were not consistent with the current taxonomy (Table S1). In this study, it was noticed that strain Q-426 was in the same clade with $B$. velezensis GB03, B. velezensis YJ11-1-4 and B. velezensis IT45 (Fig. 2). Furthermore, strains in the same clade with Q-426 on the phylogenic tree reported in a previous paper [30] have been updated to $B$. velezensis. Therefore, we need to perform whole-genome sequencing of Q-426 in the future to ensure its taxonomic status.

\section{Purification and characterization of the ComX pheromone of B. amyloliquefaciens Q-426}

The QS pheromone, ComX, is a strain-specific signaling oligopeptide that is modified from pro-ComX by the corresponding protein ComQ. The ComX pheromone has a unique modified tryptophan (W) residue with a geranyl group or a farnesyl group at the 3-position of its indole ring, resulting in the formation of a tricyclic structure. The structural variability of ComX also is related to the sequences of the peptide backbones $[14,15,17,22,38]$. As shown in Fig. 3A, conservation appears to be restricted to the $\mathrm{N}$ terminus of the protein, whereas high diversity in the $\mathrm{C}$-terminus marks divergence within the pheromoneforming region (in red letters). To date, the chemical structures of ComX pheromones have been identified for only a few Bacillus strains (Fig. 3A). No chemical structures have been identified for ComX from $B$. amyloliquefaciens.

To determine the chemical structure of ComX from B. amyloliquefaciens Q-426, we co-expressed ComQ $_{\mathrm{Q} 426}$ and ComX $\mathrm{Q}_{\mathrm{Q} 26}$ in E. coli BL21(DE3) and purified the ComX $\mathrm{Q}_{\mathrm{Q} 426}$ by gradient reverse-phase HPLC (Fig. S2). MALDI-MS spectra of the fraction collected from a retention time of 34.0 min are shown in Fig. 3B and Fig. S3. This fraction contained the characteristic compound with a molecular ion $\mathrm{at} \mathrm{m} / \mathrm{z}$ $929.2068\left(\left[\mathrm{C}_{49} \mathrm{H}_{68} \mathrm{~N}_{8} \mathrm{O}_{10}\right]^{+}\right)$and other characteristic fragment ions that matched the chemical structure of an oligopeptide with six amino acids and the 3 rd residue from the $\mathrm{C}$-terminus, a Trp residue, modified with a farnesyl group (Fig. 3C).

\section{Deletion of comX decreased lipopeptide production in B. amyloliquefaciens Q-426}

To investigate the effects of the quorum sensing system on lipopeptide production, $\operatorname{com} X$ was deleted in wild-type strain Q-426, and ComQX was overexpressed in Q426 $\triangle$ comX, resulting in the gene deletion

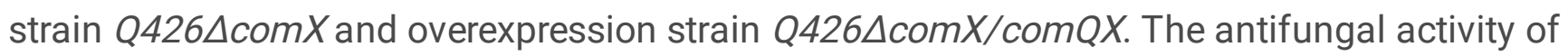
Q426 $\triangle$ com $X$ was significantly affected by the deletion, and almost no antifungal activity was observed (Fig. 4A-a), while the antifungal activity of strain Q426AcomX/comQX was restored to the same level as that of the wild-type strain (Fig. 4A-C, 4B-C). When the purified ComX $\mathrm{Q}_{\mathrm{Q}-426}$ pheromone was added to the culture medium at a final concentration of $8 \mu \mathrm{g} / \mathrm{L}$ at the early stage of the log-phase, the antifungal activity of the wild-type strain Q-426 was significantly improved (Fig. 4A-d). Also, it was observed that the antifungal activity was restored by adding extra $\mathrm{ComX}_{\mathrm{Q}-426}$ (at a final concentration of $8 \mu \mathrm{g} / \mathrm{L}$ ) during the culture of Q426AcomX. (Fig. 4A-e and 4B-e).

The previous results revealed that the Q-426 strain could produce a variety of cyclic lipopeptide compounds which were identified as C-14, C-15, C-16 bacillomycin D, C-15, C-16, C-17 fengycin A, and C- 
17 fengycin B [30]. Peak 1 and peak 2 in Fig. 4B were confirmed to be C-15 bacillomycin D, and C-16 bacillomycin D, respectively. As shown in Fig. 4B, after the addition of the purified Com $\mathrm{X}_{\mathrm{Q}-426}$ pheromone, the yield of lipopeptide production, especially, the yields of C-15 bacillomycin D (peak 1) and C-16 bacillomycin D (peak 2), were significantly increased (Fig. 4B-d and 4B-e), while there were no obvious peaks at the retention time corresponding to $\mathrm{C}-15$ bacillomycin $\mathrm{D}$ or $\mathrm{C}-16$ bacillomycin $\mathrm{D}$ in strain Q426 $\triangle$ comX (Fig. 4B-a). The above results showed that the lipopeptide synthesis is related to the quorum sensing system in strain Q-426. Collectively, these results suggested that ComX might positively affect lipopeptide synthesis, and deletion of $\operatorname{com} X$ contributed to the decrease of lipopeptide production.

\section{Effects Of Comx Deficiency On Morphology And Motility}

The wild-type strains were able to grow normally on all five media; the colony surface was rough and wrinkled; the edges were rough, and the color and size of the colonies changed according to the different media. For example, in FM medium, the colony color was dark yellow, and the folds were more obvious. In the MSgg and NB media, the colony color was white, the frill was lighter, and the edge was relatively neat (Fig. 5A and 5C). The mutant strain only formed colonies at the initial inoculation site on all the tested media and could not expand to the periphery. Except for growth on FM medium, the colonies were smooth with neat edges (Fig. 5B and 5D). The swarming ability of strain Q426 $\Delta$ comX was significantly reduced. It was difficult to expand colonies on the surface of solid medium, because the biosynthesis of lipopeptides was downregulated. As shown in Fig. 6A, when cells were gown in FM medium with a reduced agar concentration, the whole plate (bottom, wild-type strain) was quickly covered with colonies. The mutant could not move within the agar plates at any concentration of agar, and there was no significant change in the colony size. We also investigated the effects of the com $X$ gene deletion on biofilm formation. As shown in Fig. 6B, in both of FM and LB media, the mutant could not form biofilms, and the bacterial cells were deposited at the bottom of the wells. Figure 7 shows the growth curves of the wild-type strain and mutant strains. As shown from the growth curve, the lag-phase of the mutant was approximately $0-3 \mathrm{~h}$ and then the cell soon entered the log-phase, while the lag-phase of the wild-type strain was 0-5 h. However, the wild-type strain had a longer log-phase than the mutant, and the final cell density of the wild-type strain was higher than that of the mutant. Therefore, the deletion of $\operatorname{com} X$ had a certain effect on the growth, but the effect was not significant.

\section{Discussions}

In this study, we identified and characterized the comQXPA gene locus of $B$. amyloliquefaciens Q-426 and found that the stop codon of comQ and the start codon of comX overlapped by one base pair.

Furthermore, we demonstrated that most of the comQXPA loci from the Bacillus genus belong to group $\mathrm{B}$ in the variations of gene overlap. Disruption of the ComPA signaling pathway in the Q-426 strain resulted in significant effects on lipopeptide production, morphology, and motility.

Recent studies have demonstrated that the ComQXPA QS system not only exists in Bacillus-related species but also in other Gram-positive species such as Lysinbacillus, Geobacillus, and Anoxybacillus 
[19]. In Bacillus, the ComQXPA QS system exhibits striking polymorphism and is coupled to the bacterial pherotypes, which are defined as groups of bacteria that are able to communicate through com $X$ pheromones $[14,15,17,38,39]$. The evolutionary history of the comQXPA locus and the interrelation between the structural characteristics of the comQXPA locus and its ecological functions are still unclear. In this study, we analyzed 40 comQXPA sequences from completely annotated Bacillus genomes. Most of the comQXPA loci from the Bacillus genus belong to group $B$, in which the $\operatorname{com} Q$ and $\operatorname{com} X$ genes overlap, and the number of overlapping base pairs varied from 1 to 49 base pairs. From these results, although we cannot see any corresponding relation between species with overlapping type, the number of overlaps was the same within the strains located in the same clade. This suggests that overlapping reading frames in the comQXPA locus may result in more efficient or individual transcriptional control and may be associated with the evolution of specific biological functions.

Bacillomycin $D$, one member of the iturinic lipopeptide family, has strong antifungal activity, especially against filamentous fungi. Iturinic lipopeptides are mainly produced by members of the $B$. subtilis group through non-ribosomal synthetases machinery, and they exhibit strong diversity within the $B$. subtilis species group; some lipopeptides are only produced by one species, whereas certain others can be produced by up to three species [40]. The chemical principles for the biosynthesis of iturinic lipopeptide are also largely characterized [41, 42], but little is known about the regulatory mechanisms that control the expression of the iturinic lipopeptide. To date, the biosynthesis mechanisms and expression regulation mechanisms of lipopeptides except surfactin have not been extensively studied. Surfactin biosynthesis is regulated by the ComQXPA QS system in B. subtilis. Upon reaching a threshold concentration, ComX activates ComP autophosphorylation, and then ComP P transfers the phosphoryl group to ComA [25],which controls the expression of srfA encoding a very large protein complex for surfactin production that contributes to swarming motility and biofilm formation [13, 35, 38, 43-48]. Spacapan et al. also found that exoproteases required for biofilm formation are regulated by the ComQXPA system through ComX induction in B. subtilis [9]. In B. amyloliquefaciens FZB42, it has been shown that bacillomycin $\mathrm{D}$ is under direct transcriptional control of DegU, and DegQ and ComA also are required for the full transcriptional activation [49].

In our previous studies, we demonstrated that $B$. amyloliquefaciens $\mathrm{Q}-426$ can produce seven lipopeptides (C-14, C-15, and C-16 bacillomycin D; C-15, C-16, and C-17 fengycin A; C-17 fengycin $B$ ), among which the levels of $C-15$ and $C-16$ bacillomycin $D$ were higher than others $[29,30,50]$. To investigate whether the ComQXPA QS system is involved in regulating the lipopeptide synthesis in $B$. amyloliquefaciens Q-426, we disrupted the ComQXPA QS system through constructing the Q426 1 comX mutant. Our results revealed that the ComQXPA QS system might be involved in regulating bacillomycin D production in $B$. amyloliquefaciens Q-426. Interestingly, knocking out the comX gene does not affect the growth of the bacteria, but the growth capacity of the Q426AcomX mutant in liquid medium was slightly lower than that of the wild-type strain. However, the Q426AcomX lost its swimming ability, was unable to form colonies when spread on a solid surface, and could not form biofilms on the interface between the gas and liquid medium. Unfortunately, we did not confirm the direct effects of the ComQXPA QS system or ComA on lipopeptide synthesis, and this is our immediate goal. It is widely known that 
lipopeptides exhibit strong antifungal activities, but their other physiological roles in Bacillus cells are still unclear. We speculate that the ComQXPA QS system of strain Q-426 modulates some physiological and biochemical properties by regulating the biosynthesis of lipopeptides. These lipopeptide compounds themselves may also be signal molecules, and each lipopeptide has its own specific biological regulatory circuit. In a recent study, $\mathrm{Xu}$ and co-researchers pointed out that bacillomycin $\mathrm{D}$ is a signal that promotes biofilm development of $B$. velezensis SQR9 [51].

B. amyloliquefaciens $\mathrm{Q}-426$ produces an oligopeptide pheromone, Com $\mathrm{X}_{\mathrm{Q}-426}$, that inducts its $\mathrm{QS}$ system. Com $\mathrm{X}_{\mathrm{Q}-426}$ consists of six amino acids that contain a modified tryptophan residue located at the third position from the $\mathrm{C}$-terminus, resulting in the formation of a tricyclic scaffold with a pyrrolidine ring (Fig. 3C). ComX pheromones from different Bacillus strains exhibit unique amino acid sequences and residues, indicating that each bacterium has its own oligopeptide language for activating QS signaling. The linear oligopeptide (GGDWKY) without modification of the tryptophan residue with farnesyl did not activate QS signaling in the Q426AcomX strain to produce lipopeptides such as bacillomycin D (Fig. S4). This result was in close agreement with other research in that the tricyclic scaffold of the tryptophan residues in the ComX pheromone is critical to activating kinase activity of the receptor protein ComP, but there is no effect on the recognition of the receptor with pheromone molecules [17, 21, 23-25]. Further research should clarify how the sequence of amino acids of $\mathrm{ComX}_{\mathrm{Q}-426}$ contributes to the specific recognition of Com $\mathrm{X}_{\mathrm{Q}-426}$ by the receptor protein ComQ $_{\mathrm{Q}-426}$.

For Bacillus strains, it is now estimated that at least $5 \%-10 \%$ of the genome is devoted to the production of antimicrobial compounds $[45,52]$. These compounds are mainly antimicrobial cyclic peptides containing D-amino acids and hydrophobic residues. At present, studies of lipopeptides mainly focus on their physicochemical properties and biological activities, but further studies of their biological functions and the regulatory mechanisms of their biosynthesis are needed. The understanding of such biological functions and the molecular regulatory mechanism of biosynthesis will provide a theoretical basis for constructing a high-yielding lipopeptide-producing strain. This will help us to better understand the molecular mechanisms of secondary metabolism in Bacillus and its environmental adaptations.

\section{Conclusion}

In this study, we identified and characterized the comQXPA gene locus of $B$. amyloliquefaciens Q-426 and analysed 40 comQXPA sequences from completely annotated Bacillus genomes. Most of the comQXPA loci from the Bacillus genus belong to group $B$, in which the $\operatorname{com} Q$ and $\operatorname{com} X$ genes overlap and the number of overlapping base pairs varied from 1 to 49 base pair overlaps. We identified the chemical structure of ComX $\mathrm{X}_{\mathrm{Q}-426}$ that consists of six amino acids that contain a modified tryptophan residue located at the third position from the C-terminus, resulting in the formation of a tricyclic scaffold with a pyrrolidine ring. We found that strain Q426 $\Delta$ comX exhibited significantly reduced antifungal ability, almost no antifungal activity was observed, while the antifungal activity of strain Q426/comX could be restored to the same level as that of the wild-type strain. Moreover, the antifungal activity of $B$. 
amyloliquefaciens $\mathrm{Q}-426$ was significantly improved by adding the Com $\mathrm{X}_{\mathrm{Q}-426}$ pheromone to the culture medium. Interestingly, knocking out the comX gene did not affect bacterial growth. However, the $Q-426$ $\triangle \operatorname{com} X$ mutant lost swimming ability, was unable to form colonies when spread in on solid surface, and could not form biofilms on the interface between the gas and liquid medium. These funding suggest that the production of lipopeptides is controlled by the ComPA signalling pathway and relate to the swarming ability and biofilm formation in B. amyloliquefaciens Q-426.

\section{Methods}

\section{Bacterial strains, plasmids, media and culture conditions}

B. amyloliquefaciens Q-426 (CCTCC M2010237) was isolated from compost samples in Dalian, China. Escherichia coli DH5a and E. coli BL 21(DE3) were used in genetic experiments. Luria-Bertani (LB) medium comprised ( $\mathrm{g} / \mathrm{L}$ ) peptone (10.0), yeast extract (5.0) and $\mathrm{NaCl}$ (5.0). FM medium comprised ( $\mathrm{g} / \mathrm{L}$ ) tryptone (12.4), glucose (20), $\mathrm{NaCl}(5.0), \mathrm{K}_{2} \mathrm{HPO}_{4}(1.5), \mathrm{MnSO}_{4} \cdot 2 \mathrm{H}_{2} \mathrm{O}(0.04), \mathrm{FeSO}_{4} \cdot 7 \mathrm{H}_{2} \mathrm{O}$ (1.7), and $\mathrm{MgCl}_{2} \cdot 6 \mathrm{H}_{2} \mathrm{O}$ (1.2), $\mathrm{pH}$ 7.0. NB medium comprised (g/L) tryptone (10), beef powder (3.0), and $\mathrm{NaCl}$ (5.0), $\mathrm{pH}$ 7.2. MSgg medium comprised $5 \mathrm{mM}$ potassium phosphate $(\mathrm{pH} 7.0), 100 \mathrm{mM}$ morpholinepropane sulfonic acid (pH 7.0), $2 \mathrm{mM} \mathrm{MgCl}_{2}, 700 \mu \mathrm{M} \mathrm{CaCl}_{2}, 50 \mu \mathrm{M} \mathrm{MnCl}_{2}, 50 \mu \mathrm{M} \mathrm{FeCl}_{3}, 1 \mu \mathrm{M} \mathrm{ZnCl}_{2}, 2 \mu \mathrm{M}$ thiamine, $0.5 \%$ glycerol, $0.5 \%$ glutamate, tryptophan $(50 \mu \mathrm{g} / \mathrm{mL})$, and phenylalanine $(50 \mu \mathrm{g} / \mathrm{mL})$. BGM medium comprised LB medium plus $0.15 \mathrm{M}$ ammonium sulfate, $100 \mathrm{mM}$ potassium phosphate $(\mathrm{pH} 7.0)$, $34 \mathrm{mM}$ sodium citrate, $1 \mathrm{mM} \mathrm{MgSO}_{4}$, and $0.1 \%$ glucose. $\mathrm{M} 9$ medium comprised (g/L) glucose (5.0), yeast extract (4.0), $\mathrm{NH}_{4} \mathrm{NO}_{3}$ (1.0), $\mathrm{NaCl}(0.5), \mathrm{K}_{2} \mathrm{HPO}_{4}$ (1.5), $\mathrm{KH}_{2} \mathrm{PO}_{4}$ (0.5), and $\mathrm{MgSO}_{4}$ (0.2). Solid medium was obtained by adding $15 \mathrm{~g} / \mathrm{L}$ agar to the liquid medium. All strains were grown at $37^{\circ} \mathrm{C}$. Unless otherwise indicated, the final concentrations of antibiotics were as follows (mg/L): ampicillin (Amp), 50 and kanamycin (Kan), 30.

\section{Dna Manipulation Techniques}

Oligonucleotide synthesis and DNA sequencing were performed by Sangon Biotech Co., Ltd. (Shanghai, China). The isolation and manipulation of recombinant DNA were carried out as described previously [53]. All enzymes were commercial preparations. Phusion DNA high-fidelity polymerase was purchased from NEB (Shanghai, China). B. amyloliquefaciens transformations were performed by artificially inducing genetic competence [54].

\section{Cloning and sequence analysis of QS-related genes ( comQXPA )}

Genomic DNA of strain Q-426 was isolated from the bacterial culture using the Bacterial DNA Isolation Kit (Foregen, Beijing, China). To amplify the whole gene of the comQXPA locus of B. amyloliquefaciens Q426, the upstream and downstream primers (com_F and com_R; the location is indicated in Fig. 1A in red) were designed from the $\operatorname{deg} Q$ and luxR gene sequences, which are located upstream of $\operatorname{com} Q$ and 
downstream of comA, respectively. PCR amplification was employed in a $50 \mu \mathrm{L}$ final volume using the following program: at $95^{\circ} \mathrm{C}$ for $5 \mathrm{~min} ; 30$ cycles of denaturation at $95^{\circ} \mathrm{C}$ for $1 \mathrm{~min}$, annealing at $58^{\circ} \mathrm{C}$ for $1 \mathrm{~min}$ and extension at $72{ }^{\circ} \mathrm{C}$ for $1 \mathrm{~min}$; final extension at $72{ }^{\circ} \mathrm{C}$ for $10 \mathrm{~min}$. PCR products were detected by $1 \% \mathrm{~W} / \mathrm{V}$ agarose gel electrophoresis at $100 \mathrm{~V}$ for $30 \mathrm{~min}$. The nucleotide sequences of amplified PCR fragments were determined by Sangon Biotech Co., Ltd. using an ABI DNA Sequencer (3730XL, USA) and then submitted to NCBI GenBank for BLAST analysis (http://www.ncbi.nlm.nih.gov/BLAST, GenBank accession no. MF579444).

The nucleotide sequence of the amplified PCR fragment was then compared with the corresponding gene clusters from strains with homologous genes from other Bacilli, which were obtained from the NCBI nucleotide/protein database. Alignments for the phylogenetic tree were made using ClustalX-1.81 software. Phylogenetic analyses were conducted using MEGA version 7.0.20 for Neighbor-Joining and ME analyses using the Kimura 2-parameter model. Measures of bootstrap support for internal branches were obtained from 1000 pseudoreplicates.

Deletion of the comX gene in B. amyloliquefaciens strain Q-426

The left flanking (LF) region (85 bp), kan region (813 bp), and right flanking (RF) region (80 bp) were amplified from the genomic DNA of Q-426 and pET-28-(a+) using the primer pairs of comX_F/comXI_R, kan_F/kan_R and comXП_F/comXП_R (Table 1, Fig. 1B), respectively. These three fragments were fused using overlap PCR in the order of LF, KAN, and RF. The resulting 1.0-kb comX deletion amplicon $(1 \mu \mathrm{g})$ was directly transformed $(25 \mu \mathrm{F}, 600 \Omega)$ into strain Q-426, and 53 colonies were obtained from LB plates containing Kanamycin $\left(30 \mu \mathrm{g} \cdot \mathrm{mL}^{-1}\right)$. Three colonies confirmed by PCR were cultivated to an $\mathrm{OD}_{600}$ of 1.0 without Kan, and a 100- $\mu \mathrm{L}$ aliquot of a 10-fold dilution of the cultures (approximately $10^{5}$ cells) was plated on LB-Kan medium. Mutants growing on LB-Kan were further confirmed by PCR and DNA sequencing. Mutants selected were retreated on LB-Kan plates for 20 times to confirm their stability. 
Table 1

Plasmids and strains used in this study

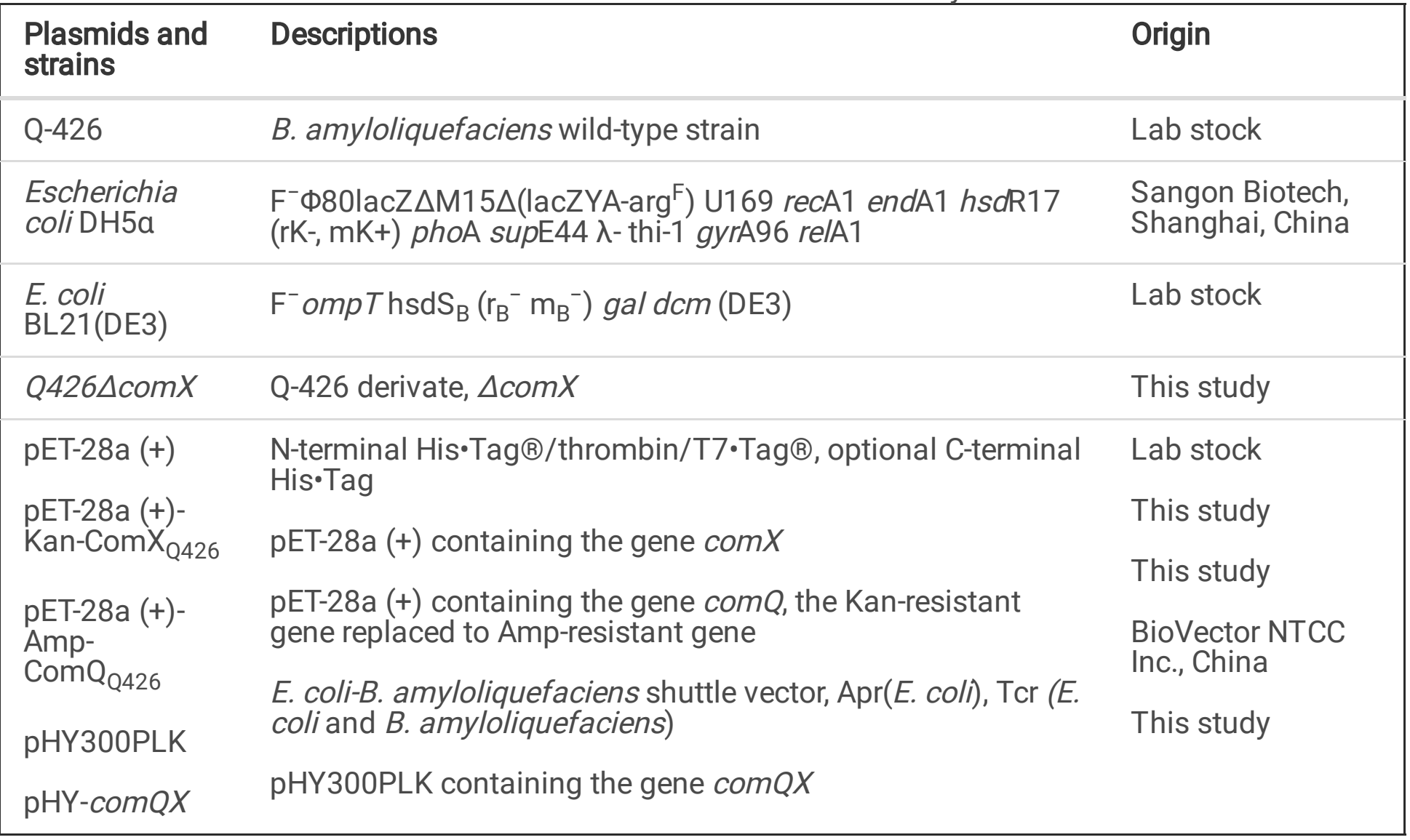

The ComQX expression vector was constructed according to a previously reported method [55]. Briefly, the gene comQX was amplified by the corresponding primers (ComQX_F/R, Table 2). Then, the fragment was inserted into pHY300PLK, a shuttle vector for $E$ coli and B. subtilis (BioVector NTCC Inc., China), at the restriction enzyme sites $\mathrm{BamHI} / \mathrm{Xbal}$, resulting in the comQX expression vector named as $\mathrm{pHY}$ comQX. The vectors $\mathrm{pHY}$-comQX were first electroporated into $E$. coli $\mathrm{DH} 5 \mathrm{a}$ to confirm and prepare an efficient expression vector, and then electroporated into Q426 $\triangle$ comX competent cells. The recombinant strains were selected by tetracycline-resistance $\left(25 \mu \mathrm{g} \cdot \mathrm{mL}^{-1}\right)$ and named $Q-426 \Delta \operatorname{com} X / \operatorname{Com} X$. 
Table 2

The primers used in this study

\begin{tabular}{|ll|}
\hline Primers names & Sequence $\mathbf{5}^{\prime} \rightarrow \mathbf{3}^{\prime}$ \\
\hline com_F & GTTATCTGACTTGGCGATCAGC \\
com_R & GAGAAACAACCGACTCATTACG \\
comXI_R & GGATCCATGCAGGAAATTGTAGGATACTTAACC \\
kan_F & ATGGCTCATCTAACTCCGATCAGACTAGC \\
kan_R & GATCCTGGCTGCCGATTTCGGCCTATTGG \\
comXII_F & AATCGGCAGCCAGGATCAAACAGATTGTG \\
comXII_R & GTCGACTCAATATTTCCAATCACCGCCGAAAG \\
RF-ComX_F & CATCATCATCATCATCACAGCAGCATGCAGGAAATTGTAGGATACTTAACC \\
RF-ComX_R & GGCTTTGTTAGCAGCCGGATCTCAGTTAATATTTCCAATCACCGCCGAAAG \\
ComQ_F & CGGGATCCATGTGTTTAGATGCCGAAACG \\
ComQ_R & CGGTCGACGCTAACTCCGATCAGACTAGC \\
ComQX_F & CGCGGATCCATGTGTTTAGATGCCGAAACG \\
ComQX_R & CCGGAATTCTCAATATTTCCAATCACCGC \\
\hline
\end{tabular}

\section{Colony Morphology And Growth Curve Of Strain Q426 $\Delta$ comx}

Wild-type B. amyloliquefaciens Q-426 or the Q426 4 comX mutants were grown overnight on LB agar plates, resuspended in PBS $\left(\mathrm{OD}_{600}=0.2\right)$ and sonicated for $12 \mathrm{~s}$ with 1-s on/off pulses. Two microliters of each culture were then spotted onto the center of LB, NB, FM, BGM and MSgg agar plates and incubated at $30^{\circ} \mathrm{C}$ for $48 \mathrm{~h}$.

For growth curve determination, a fresh single colony of each tested strain was inoculated into $200 \mathrm{~mL}$ FM liquid medium in a 1-L flask with $1 \%$ inoculation and incubated at $30^{\circ} \mathrm{C}$ and $180 \mathrm{rpm}$. A certain amount of liquid culture was taken from the flask, and the $\mathrm{OD}_{600}$ was measured every hour on the first day and then measured every 4 or $8 \mathrm{~h}$ after reaching a stable period. The average value was measured three times for each group of data.

\section{Overexpression Of Comq And/or Comx}


The recombinant plasmids pET-28a (+)-Kan-ComX and pET-28a (+)-Amp-ComQ were separately or simultaneously transformed into E. coli BL21(DE3) to express ComX, ComQ and ComX/ComQ, a protein with an His-tag at its N-terminal (Fig. S2). E. coli BL21(DE3) cells harboring pET-28a(+)-Kan-ComX or pET28a(+)-Amp-ComQ or both pET-28a(+)-Kan-ComX and pET-28a(+)-Amp-ComQ were grown on LB medium (containing the indicated antibiotics) at $37^{\circ} \mathrm{C}$ and $180 \mathrm{rpm}$ until the $\mathrm{OD}_{600}$ reached approximately 0.7 , and gene expression was induced by adding isopropyl $\beta$-d-1-thiogalactopyranoside (IPTG) at a final concentration of $0.1 \mathrm{mM}$. The expression of ComQ and ComX were confirmed by SDS-PAGE.

\section{Western Blotting}

Proteins were separated by $12 \%$ SDS-PAGE and transferred onto polyvinylidenedifluoride (PVDF) membranes (Bio-Rad, Hercules, CA, USA) by electroblotting for Western blot analysis. The mouse anti-Histag antibody (Sangon Biotech Co., Ltd.) was used as the primary antibody at a 1:2000 dilution in blocking solution ( $2 \%$ no protein blocking solution, Sangon Biotech Co., Ltd.). The rabbit-anti-mouse horseradish peroxidase (HRP)-conjugated secondary antibody (Sangon Biotech Co., Ltd.) was used at a dilution of 1:3000. Protein bands were detected on photographic films using an enhanced chemiluminescent substrate. The EasySee ${ }^{\circledR}$ Western Marker (TRAN Co., Beijing, China) was used for calculating molecular weights of proteins after western blotting.

\section{Pheromone Overproduction And Identification}

The E. coli ComX-producing strain that co-expressed comX and comQ was grown overnight in completed M9 medium supplemented with a mixture of amino acids (Phe, Ser, His, Met, at $40 \mathrm{ug} / \mathrm{mL}$; and Gln at $0.4 \mathrm{mg} / \mathrm{mL})$, kanamycin $(30 \mu \mathrm{g} / \mathrm{mL})$, and ampicillin $(50 \mu \mathrm{g} / \mathrm{mL})$. At the stationary phase, $20 \mathrm{~mL}$ of the pre-culture was added to $1980 \mathrm{~mL}$ of the supplemented M9 medium to make a 2-L bacterial culture, which was then incubated at $37^{\circ} \mathrm{C}$ and $110 \mathrm{rpm}$ for $8 \mathrm{~h}$. The expression of the comQX gene was induced with $0.1 \mathrm{mM} \mathrm{IPTG}$ at $37^{\circ} \mathrm{C}$ and $110 \mathrm{rpm}$ overnight. The culture broth was centrifuged for $10 \mathrm{~min}$ at 8000 $\times g$. The supernatant was filtered through a $0.22 \mu \mathrm{m}$ filter, and $\mathrm{CH}_{3} \mathrm{CN}$ was added to a final concentration of $20 \%$. The filtrate was loaded onto a column packed with $\mathrm{HZ}$ resin (Huazhen Tech, Shanghai, China) that was equilibrated with $10 \% \mathrm{CH}_{3} \mathrm{CN}$ and 0.1 TFA. The column was eluted with a step gradient of $\mathrm{CH}_{3} \mathrm{CN}(20,40,50,65$, and $80 \%$ in $0.1 \%$ aqueous TFA). The $50 \%$ elute containing ComX was immediately neutralized with aqueous ammonia, concentrated with a rotary evaporator, and dried with a freeze dryer. The dried extract was analyzed through HPLC (1260 Infinity, Agilent Technologies, Santa Clara, CA, USA) equipped with a C18 column (Agilent Technologies, $5 \mu \mathrm{m} \times 4.6 \times 150 \mathrm{~mm}$ ) to detect the ComX pheromone. MALDI-MS (matrix-assisted laser desorption/ionization mass spectrometry) was used to determine the mass and the amino acid sequences of the pheromone peptide present in the samples collected from HPLC.

\section{Effects of the ComX pheromone and ComX gene deletion on lipopeptide production}




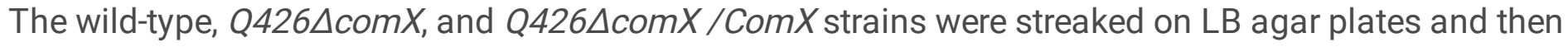

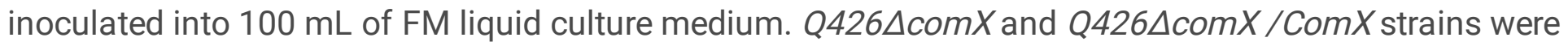
transferred to the FM liquid culture medium containing corresponding antibiotics in a $1 \%$ inoculation volume. The fermentation process was conducted at $180 \mathrm{rpm}$ for $72-76 \mathrm{~h}$ at $30^{\circ} \mathrm{C}$. Lipopeptide productivity and antifungal activity assays were evaluated according to a previous study [31].

\section{Declarations}

\section{Acknowledgments}

We would like to thank Accdon-LetPub (http://www.letpub.com) for their English language editing.

\section{Authors' contributions}

$\mathrm{CQ}, \mathrm{SF}, \mathrm{WZ}$, and PZ designed the experiments. BG, JL, WZ, XS, and JL performed the experiments with assistance from RF. CQ, PZ, and LZ wrote the manuscript.

\section{Funding}

This work was supported by the National Natural Science Foundation of China (Grant No. 21272031, 21372037), the Liaoning Natural Science Foundation Joint Fund project (2020-MZLH-07), and the Dalian High Level Talents Innovation Support Plan (2019CT09).

\section{Availability of data and materials}

Sequence data is available in GenBank (GenBank accession no. MF579444). All datasets generated for this study are included in the article/Supplementary Material.

\section{Ethics approval and consent to participate}

Not applicable.

\section{Consent for publication}

Not applicable.

\section{Competing interests}

The authors declare no conflict of interest. The authors declare that the research was conducted in the absence of any commercial or financial relationships that could be construed as a potential conflict of interest.

\section{References}


1. Caulier S, Nannan C, Gillis A, Licciardi F. Overview of the antimicrobial compounds produced by members of the Bacillus subtilis Group. Front Microbiol. 2019;10:302.

2. Liu X, Min Y, Wang K, Wan Z, Zhang Z. Draft genome sequence of Bacillus amyloliquefaciens HB-26. Stand Genom Sci. 2014;9(3):775-782. doi:10.4056/sigs.4978673

3. Wu LM, Wu HJ, Qiao JQ, Gao XW, Borriss R. Novel routes for improving biocontrol activity of Bacillus based bioinoculants. Front Microbiol. 2015; 6:1395.

4. Arguelles-Arias A, Ongena M, Halimi B, Lara Y, Brans A, Joris B, Fickers P. Bacillus amyloliquefaciens GA1 as a source of potent antibiotics and other secondary metabolites for biocontrol of plant pathogens. Microb Cell Fact. 2009;8:63.

5. Junaid JM, Dar NA, Bhat TA, Bhat AH, Bhat A. Commercial biocontrol agents and their mechanism of action in the management of plant pathogens. Int J Mod Plant Anim Sci. 2013;1(2):39-57.

6. Sun J, Qian SQ, Lu J, Liu YN, Lu FX, Bie XM, Lu ZX. Knockout of rapC improves the Bacillomycin D yield based on de novo genome sequencing of Bacillus amyloliquefaciens fmbJ. J Agric Food Chem. 2018; 66(17):4422-4430. doi:10.1021/acs.jafc.8b00418

7. Bassler BL, Losick R. Bacterially speaking. Cell. 2006; 125(2):237-246. doi:10.1016/j.cell.2006.04.001

8. Kalamara M, Spacapan M. Social behaviours by Bacillus subtilis: quorum sensing, kin discrimination and beyondd. Mol Microbiol. 2018; 110(6):863-878.

9. Spacapan M, Danev T. ComX induced exoproteases degrade ComX in Bacillus subtilis. Front Microbiol. 2018;9:105.

10. Whiteley M, Stephen P. Progress in and promise of bacterial quorum sensing research. Nat Publ Gr. 2017; 551(7680): 313-320.

11. Comella N, Grossman AD. Conservation of genes and processes controlled by the quorum response in bacteria: characterization of genes controlled by the quorum-sensing transcription factor ComA in Bacillus subtilis. Mol Microbiol. 2005;57(4):1159-1174.

12. Bareia T, Pollak S, Eldar A. Self-sensing in Bacillus subtilis quorum-sensing systems. Nat Microbiol. 2018; 3(1):83-89.

13. Oslizlo A, Stefanic P, Dogsa I,Mandic-mulec I. Private link between signal and response in Bacillus subtilis quorum sensing. PNAS U.S.A. 2014; 111(4):1586-1591.

14. Oslizlo A, Stefanic P, Vatovec S, Glaser B, Rupnik M. Exploring ComQXPA quorum-sensing diversity and biocontrol potential of Bacillus spp. isolates from tomato rhizoplane. Microbiol Biotech. 2015;8(3): 527-540.

15. Tran LP, Nagai T. Divergent structure of the ComQXPA quorum-sensing components: molecular basis of strain-specific communication mechanism in Bacillus subtilis. Mol Microbiol. 2000; 37(5):11591171.

16. Wu Q, Zhi Y, Xu Y. Systematically engineering the biosynthesis of a green biosurfactant surfactin by Bacillus subtilis 168. Metab Eng. 2019;52:87-97. 
17. Ansaldi M, Marolt D, Stebe T, Mandic-mulec, I. \& Dubnau, D. Specific activation of the Bacillus quorum-sensing systems by isoprenylated pheromone variants. Mol Microb. 2002;44:1561-1573.

18. Esmaeilishirazifard E, Vizio DD, Moschos SA, Keshavarz T. Genomic and molecular characterization of a novel quorum sensing molecule in Bacillus licheniformis. AMB Express. 2017; 7(1):78.

19. Dogsa I, Choudhary KS, Marsetic Z, Hudaiberdiev S, Vera R. ComQXPA quorum sensing systems may not be unique to Bacillus subtilis: A census in prokaryotic genomes. PLoS One. 2014;9(5): e96122.

20. Huber M, Faure G, Laass S, Kolbe E, Seitz K, Wehrheim C, Yuri I, Wolf EV, Koonin \& Jörg S. Translational coupling via termination-reinitiation in archaea and bacteria. Nat Commun. 2019;10(1):4006.

21. Ansaldi M, Dubnau D. Diversifying selection at the Bacillus quorum-sensing locus and determinants of modification specificity during synthesis of the ComX pheromone. J Bacteriol. 2004;186:15-21.

22. Hayashi S, Usami S, Nakamura Y, Ozaki K, Okada M. Identification of a quorum sensing pheromone posttranslationally farnesylated at the internal tryptophan residue from Bacillus subtilis subsp. natto. Biosci Biotech Biochem. 2015;79:1567-1569.

23. Okada M, Sato I, Cho SJ, Iwata H, Nishio T, Dubnau D, Sakagami Y. Structure of the Bacillus subtilis quorum-sensing peptide pheromone ComX. Nat Chem Biol.2005; 1(1):23-24.

24. Okada M, Sugita T, Abe I. Posttranslational isoprenylation of tryptophan in bacteria. J Org Chem. 2017;22:338-346.

25. Schneider KB, Palmer TM, Grossman AD. Characterization of comQ and comX, two genes required for production of ComX pheromone in Bacillus subtilis. J Bacteriol. 2002; 184:410-419.

26. Tsuji F, Kobayashi K, Okada M, Yamaguchi H, Ojika M, Sakagami Y. The geranyl-modified tryptophan residue is crucial for ComX RO-E-2 pheromone biological activity. Bioorg. Med Chem Lett.2011;21: 4041-4044.

27. Tsuji F, Ishihara A, Kurata K, Nakagawa A, Okada M, Kitamura S, Kanamaru K, Masuda Y, Murakami K, Irie K. Geranyl modification on the tryptophan residue of ComX RO-E-2 pheromone by a cell-free system. FEBS Lett. 2012;586(2):174-179.

28. Tsuji F, Ishihara A, Nakagawa A, Okada M, Kitamura S, Kanamaru K. Lack of the consensus sequence necessary for tryptophan prenylation in the ComX pheromone precursor. Biosci Biotechnol Biochem.2012;76: 1492-1496.

29. Zhao PC, Quan CS, Jin LM, Wang LN, Wang JH, Fan SD. Effects of critical medium components on the production of antifungal lipopeptides from Bacillus amyloliquefaciens Q-426 exhibiting excellent biosurfactant properties. World J Microbiol Biotech. 2013;29: 401-409.

30. Zhao PC, Quan CS, Jin LM, Wang LN, Guo XJ, Fan SD. Sequence characterization and computational analysis of the non-ribosomal peptide synthetases controlling biosynthesis of lipopeptides, fengycins and bacillomycin D, from Bacillus amyloliquefaciens Q-426. Biotech Lett. 2013;35:21552163.

31. Zhao PC, Quan CS, Wang YG, Wang JH, Fan SD. Bacillus amyloliquefaciens Q-426 as a potential biocontrol agent against Fusarium oxysporum f. sp. spinaciae. J Basic Microbiol. 2013;54:448-456. 
32. Dang YL, Zhao FJ, Liu XS, Fan X, Huang R, Gao WX, Wang S, Yang C. Enhanced production of antifungal lipopeptide iturin A by Bacillus amyloliquefaciens LL3 through metabolic engineering and culture conditions optimization. Microb Cell Fact. 2019;18:68.

33. Zhi Y, Wu Q Xu Y. Genome and transcriptome analysis of surfactin biosynthesis in Bacillus amyloliquefaciens MT45. Sci Rep.2017; 7:40976.

34. Kaspar F, Neubauer P, Gimpel M. Bioactive secondary metabolites from Bacillus subtilis: A comprehensive review. J Nat Prod. 2019; 82:2038-2053.

35. Xu Z, Shao J, Li B, Yan X, Shen Q. Contribution of bacillomycin D in Bacillus amyloliquefaciens SQR9 to antifungal activity and biofilm formation. Appl Environ Microbiol. 2013;79: 808-815.

36. Branda SS, Gonzalez-Pastor JE, Dervyn E, Ehrlich SD, Losick R, Kolter R. Genes involved in formation of structured multicellular communities by Bacillus subtilis. J Bacteriol.2004;186: 3970-3979.

37. Mielich-süss, B. \& Lopez, D. Molecular mechanisms involved in Bacillus subtilis biofilm formation. Environ Microbiol. 2015;17: 555-565.

38. Stefanic P, Mandic-mulec I. Social interactions and distribution of Bacillus subtilis pherotypes at microscale. J Bacteriol. 2009;191:1756-1764.

39. Tortosa P, Logsdon L, Kraigher B, Itoh Y. Specificity and genetic polymorphism of the Bacillus competence quorum-sensing system. J Bacteriol. 2001;183: 451-460.

40. Dunlap CA, Bowman MJ, Rooney AP. Iturinic lipopeptide diversity in the Bacillus subtilis species group- important antifungals for plant disease biocontrol applications. Front Microbiol. 2019; 10:1794.

41. Duitman EH, Hamoen LW, Rembold M, Venema G, Seitz H. Saenger W, Bernhard F, Reinhardt R, Schmidt M, Ullrich C, et al. The mycosubtilin synthetase of Bacillus subtilis ATCC6633: A multifunctional hybrid between a peptide synthetase, an amino transferase, and a fatty acid synthase. Proc Natl Acad Sci. 1999;96(23):13294-13299.

42. Tsuge K, Akiyama T, Shoda M. Cloning, sequencing, and characterization of the iturin A Operon. Front Microbiol. 2001;183: 6265-6273.

43. Aleti G, Lehner S, Bacher M, Nikolic B. Surfactin variants mediate species-specific biofilm formation and root colonization in Bacillus. Environ Microbiol. 2016;18:2634-2645.

44. Bais HP, Fall R, Vivanco JM. Biocontrol of Bacillus subtilis against infection of arabidopsis roots by Pseudomonas syringae is facilitated by biofilm formation and surfactin. Plant Physiol. 2004;134: 307-319.

45. Vargas-bautista $C$, Rahlwes $K$, Straight $P$. Bacterial competition reveals differential regulation of the pks genes by Bacillus subtilis. J Bacteriol. 2014;196: 717-728.

46. Hamoen LW, Venema G, Kuipers OP. Controlling competence in Bacillus subtilis : shared use of regulators. Microbiology. 2003;149:9-17.

47. Wolf D, Rippa V, Mobare JC, Sauer P, Adlung L, Kolb P,Bischpfs IB. The quorum-sensing regulator ComA from Bacillus subtilis activates transcription using topologically distinct DNA motifs. Nucleic 
Acids Res. 2016;44: 2160-2172.

48. Zeriouh H, Vicente AD, Pérez-garcía A, Romero D. Surfactin triggers biofilm formation of Bacillus subtilis in melon phylloplane and contributes to the biocontrol activity. Environ. Microbiol. 2013;16:2196-2211.

49. Koumoutsi A, Chen X, Vater J, Borriss R. DegU and YczE Positively regulate the synthesis of bacillomycin D by Bacillus amyloliquefaciens strain FZB42†. Appl Environ Microb.2007;73: 69536964.

50. Quan CS, Liu J, Zhou W, Zheng W, Jin LM, Zhao J, Zhao PC, Fan SD. Isolation, purification and antitumor activity of bacillomycin D from Bacillus amyloliquefaciems Q-426. Chin J Biotech. 2018;34(2): 235-245.

51. Xu ZH, Mandic-Mulec I, Zhang H, Liu Y, Sun X, Feng H, Xun W, Zhang N, Shen Q, Zhang R. Antibiotic bacillomycin D affects iron acquisition and biofilm formation in Bacillus velezensis through article antibiotic bacillomycin D affects iron acquisition and biofilm formation in Bacillus velezensis through a Btr-Mediated FeuABC-dependent pathway. Cell Rep 2019;29: 1192-1202.

52. Gu Q, Yang Y, Yuan Q, Shi G, Wu L, Lou Z, Huo R, Wu HJ, Borriss R, Gao XY. Bacillomycin D produced by Bacillus amyloliquefaciens is involved in the antagonistic interaction with the plat-pathogenic fungus Fusarium graminearum. Appl Environ Microbiol.2017; 83(19): e01075-17.

53. Burby PE, Simmons LA. MutS2 Promotes homologous recombination in Bacillus subtilis. J Bacteriol. 2016;199(2): e00682-16.

54. Xue G, Johnson JS, Dalrymple BP. Methods High osmolarity improves the electro-transformation efficiency of the gram-positive bacteria Bacillus subtilis and Bacillus licheniformis. J Microbiol Method.1999;34:183-191.

55. Cai D, He P, Lu X, Zhu C, Zhu J, Zhan Y, Wang Q, Wen Z, Chen S. A novel approach to improve poly- Y glutamic acid production by NADPH Regeneration in Bacillus licheniformis WX-02. Sci Rep. $2017 ; 7: 43404$.

\section{Figures}


(A)

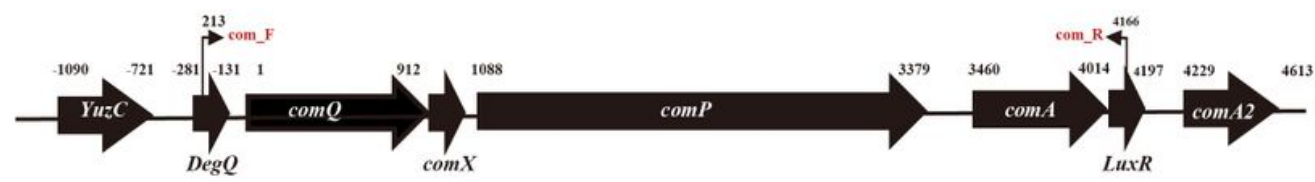

(B)

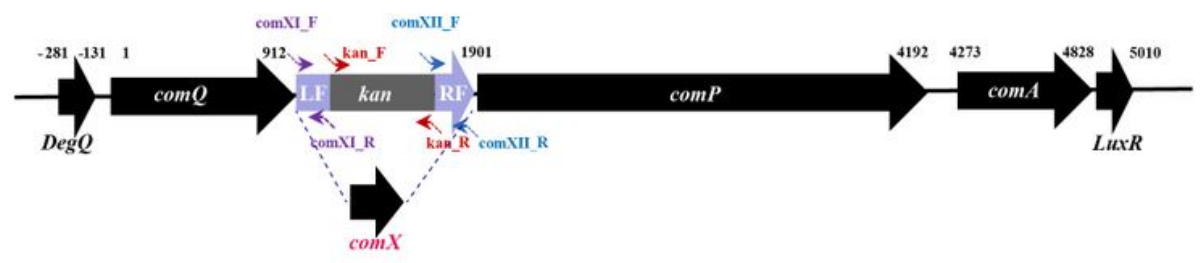

\section{Figure 1}

The comQXPA locus in B. amyloliquefaciens Q-426 and Q426 $\Delta$ comX. (A) Gene diagram of comQXPA in wild-type strain. The location of primers (com_F and com_R) are indicated in red (B) Schematic overview of the comX gene disruption strategy. The primer pairs of comX_F/comXI_R, kan_F/kan_R, and comXП_F/comXП_R are labeled in different colors. 


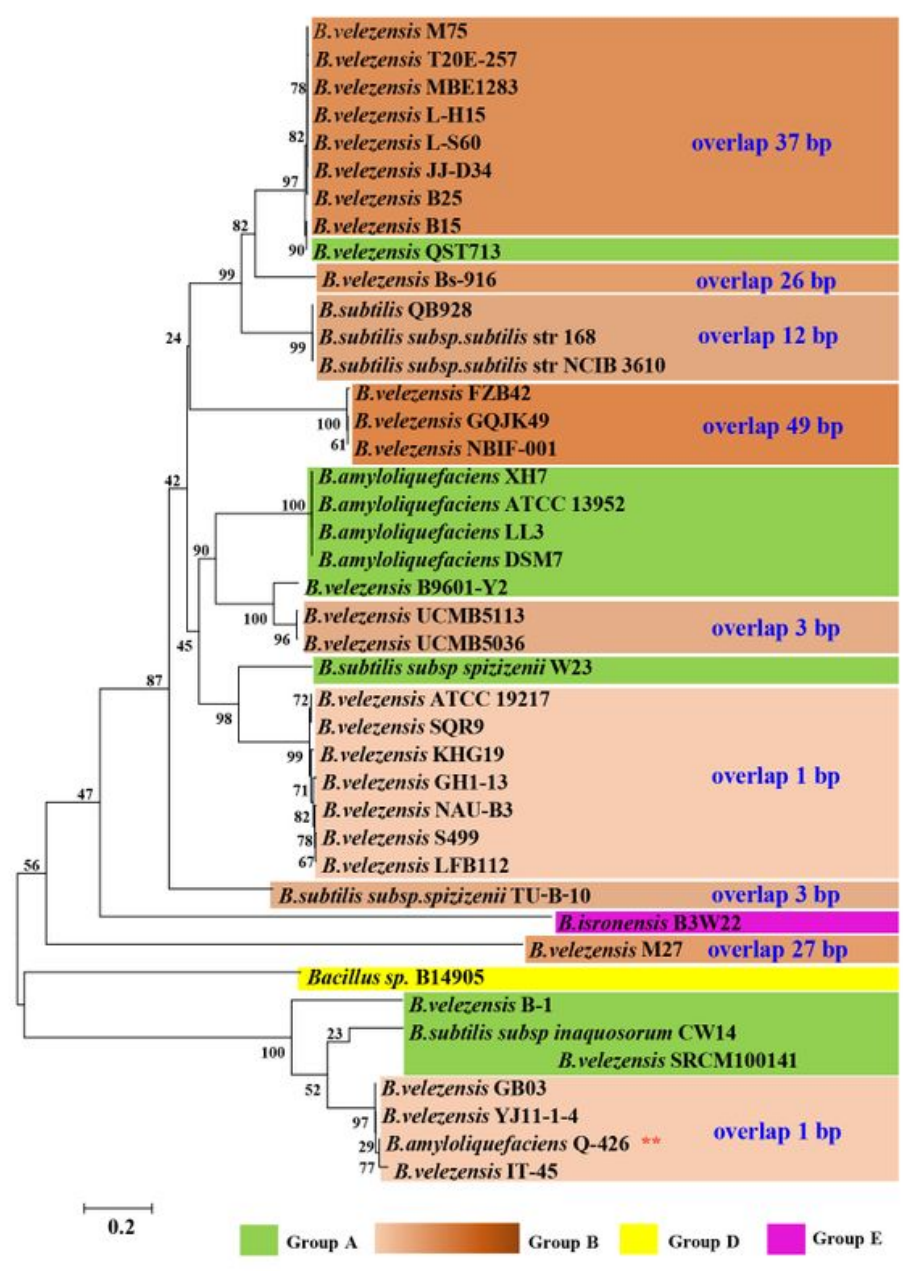

Figure 2

Neighbor-joining phylogenetic tree constructed from comQ gene. Different background color indicates different group. Green, Group A; Red, Group B, gradation of color shows variation of overlapping number of base pairs; Yellow, Group D; Pink, Group E. 
A

B. subtilis Natto $_{73} \quad$ MKHIDKIISHLVNNPEAFDQFKNGNL TLLNINEKEKKAILYAFEQGEVPRTSKWPPIEAISNFFEDDKRKSLI

B. subtilis 168 $_{55}$ MQDLINYFLNYPEALKKLKNKEACLIGFDVQETETIIKAYNDYYLADPITRQWGD

B. mojavensis RO-C-2 56

B. amyloliquefaciens $\mathrm{Q}-\mathbf{4 2 6}_{55}$

MMQDLINYFLSYPEVLKKLKNGEACLIGFSSNETETIIKAYNDYHLSSPTTREWDG

MQEIVGYLTKNPEVLNKVIEGNASLIGVSQDQTDCVINAFKGIDVISFGGDWKY

B. subtilis RO-E-2 59

B. subtilis RS-B-1 $1_{54}$

B. mojavensis RO-B-2 $2_{54}$

B. mojavensis RO-H-1 $\mathbf{1}_{54}$

MKQDMIDYLMKNPQVLTKLENGEASLIGIPDKLIPSIVDIFNKKMTLSKKCKGIFWEQ MQEMVGYLIKYPNVLREVMEGNACLLGVDKDQSECIINGFKGLEIYSMMDWHY MQEIVGYLVKNPEVLDEVMKGRASLLNIDKDQLKSIVDAFGGLQIYTNGNWVPS MQEMVGYLIKYPNVLREVMEGNACLLGVDKDQSECIINGFKGLEIYSMLDWKY

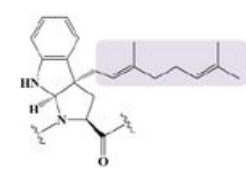

B

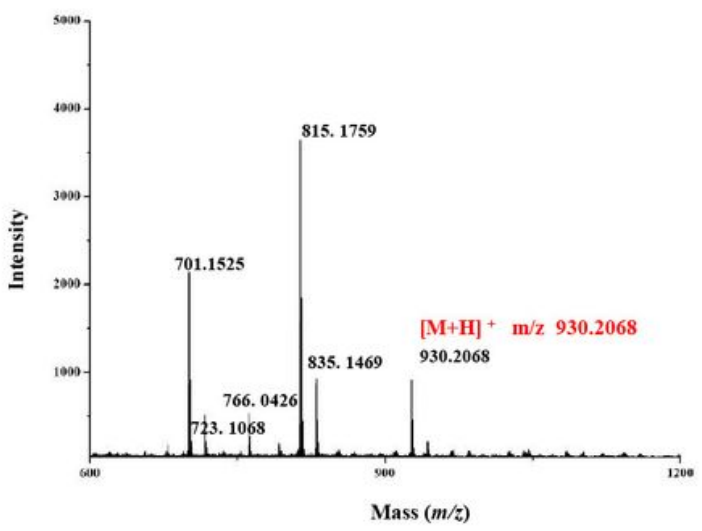

C

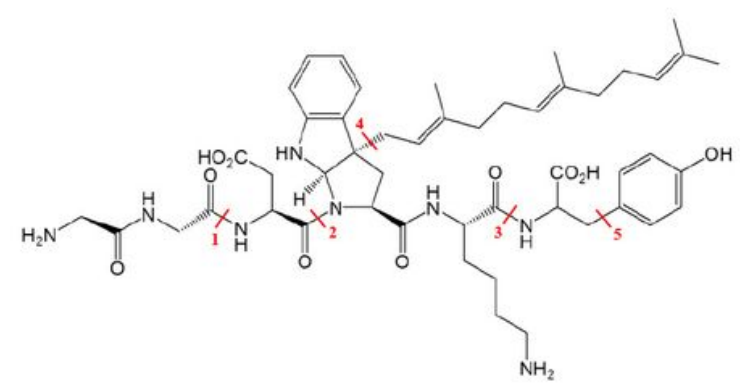

\section{Figure 3}

Identification of ComX pheromone of B. amyloliquefaciens Q-426. (A) Comparison of amino acid sequences and the modified residue of ComX pheromones. Except for the Q-426 the pre-ComX sequences are originated from previous studies.17,24 The modified tryptophan residues (asterisk) are marked with blue box. The amino acid sequence of the mature ComX is shown in red. There are two types of posttranslational isoprenylations of Com X pheromones and the isoprenyl side chains are shown in purple box. (B) MALDI-MS spectra of ComXQ426 component obtained by HPLC. (C) The chemical structure of ComXQ426. The chemical bond breaking points are shown in red. 
A
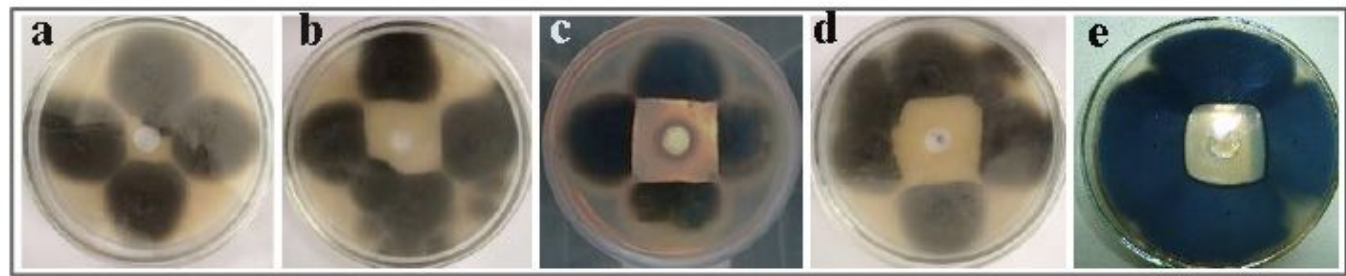

B

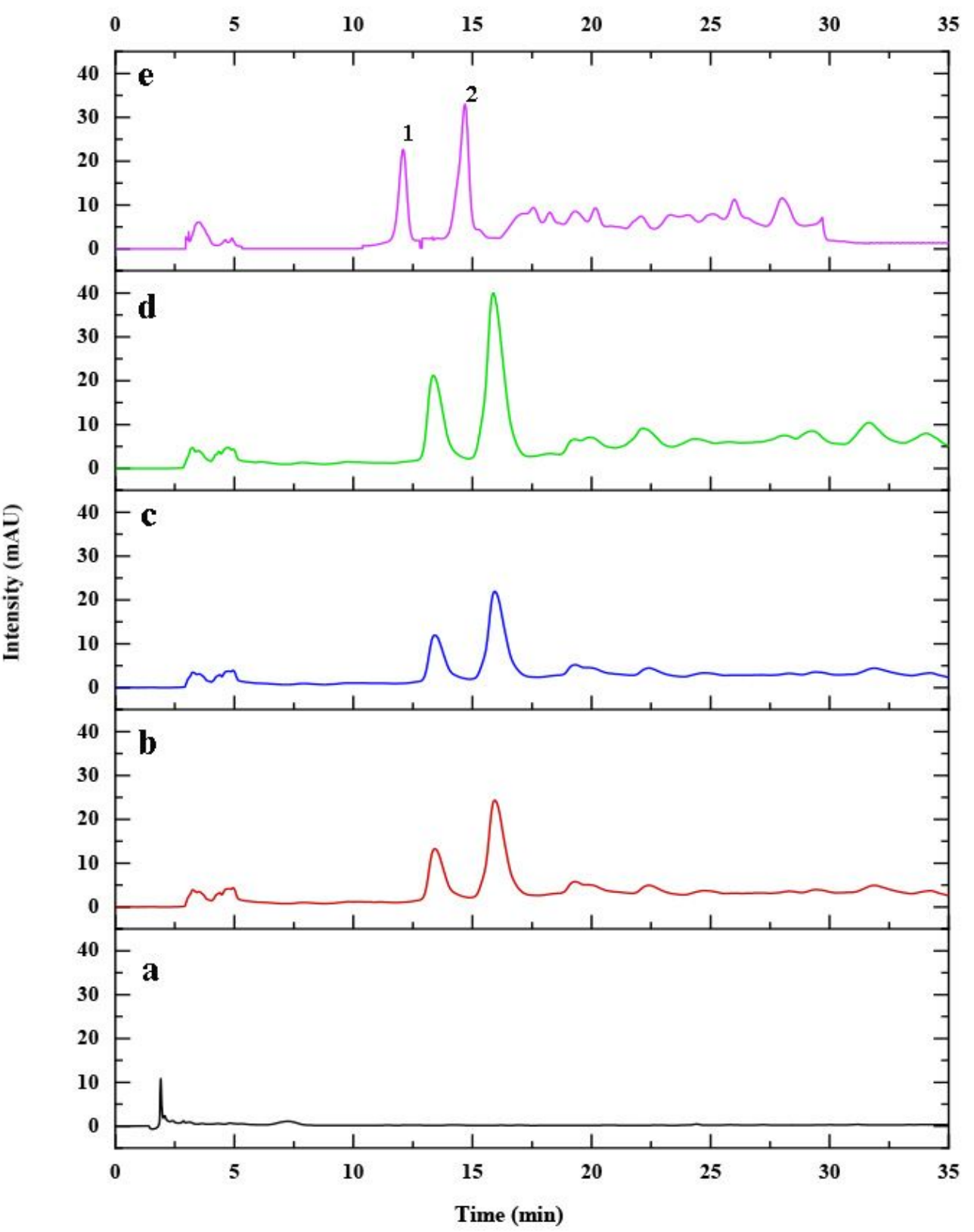

Figure 4

Effects of QS system on lipopeptide production in B. amyloliquefaciens Q-426. (A) Inhibitory activities of $100 \mu \mathrm{l}$ concentrated CFS (cell-free supernatant) on PDA medium against Curvularia lunata (Walk) Boed. All strains were cultivated in FM liquid medium for $72 \mathrm{~h}$ at $30 \mathrm{GC}$ and then the fermentation broth was condensed to $1 / 10$ before detecting the antifungal activity. (B) HPLC detection of cyclic lipopeptide from fermentation broth in different strains. a, Q426 $\Delta$ comX; b, wild-type strain; c, Q426 $\Delta$ comX/comQX囚d, wild- 
type strain + ComXQ-426. e, Q426 $\triangle$ comX+ComXQ-426. Peak 1 and peak 2 in all samples were detected as $\mathrm{C}-15$ bacillomycin $\mathrm{D}$ and $\mathrm{C}-16$ bacillomycin $\mathrm{D}$ by HPLC-MS.

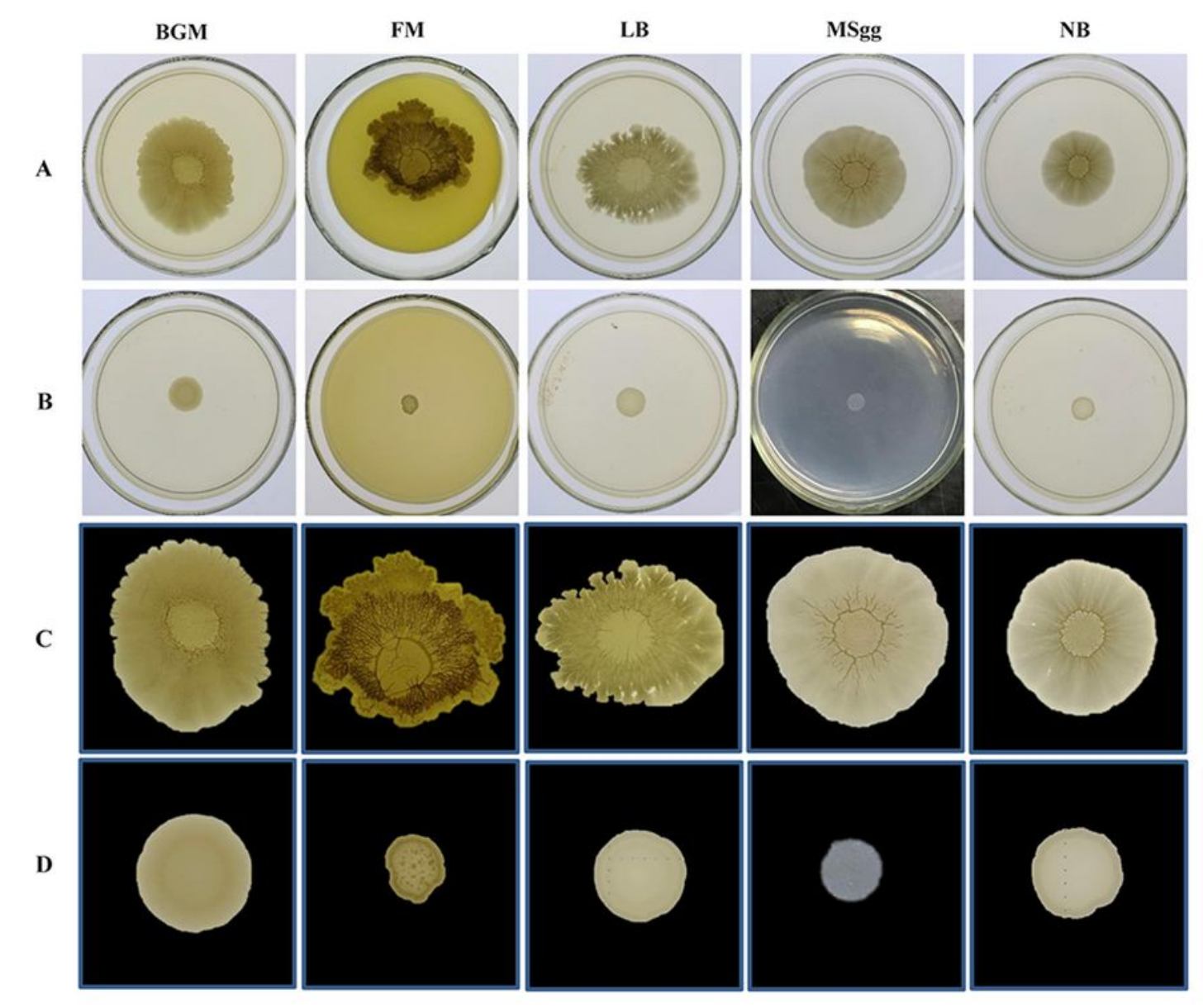

Figure 5

Colony morphology of Q426 $\Delta$ comX and its wild-type strain on different solid-media. (A) and (C), wild-type strain. (B) and (D), Q426 $\Delta$ comX. (C) and (D), Enlargement of colony image. Strains were grown in different solid media for $72 \mathrm{~h}$ at $30^{\circ} \mathrm{C}$ 
A

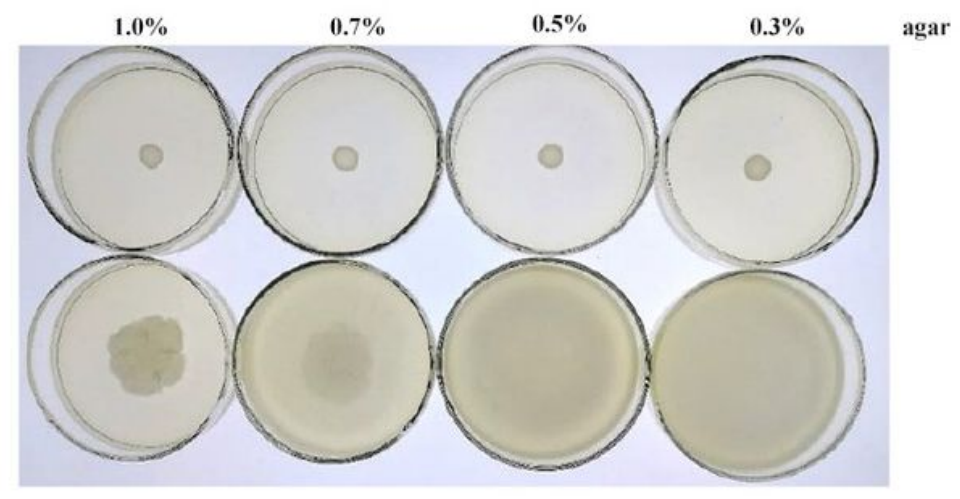

B

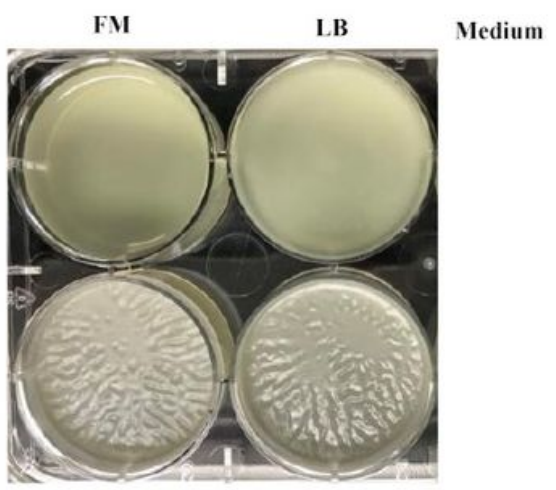

Figure 6

Biofilm formation and swarming motility of Q426 $\Delta$ comX. (A) Swarming motility. Strains were incubated on FM solid media with different concentration of agar for $72 \mathrm{~h}$ at $30^{\circ} \mathrm{C}$. (B) Biofilm formation ability. Strains were stationarity incubated in FM and LB liquid media for $72 \mathrm{~h}$ at $30^{\circ} \mathrm{C}$. Top, Q426 $\Delta$ comX; Bottom, wild-type strain.

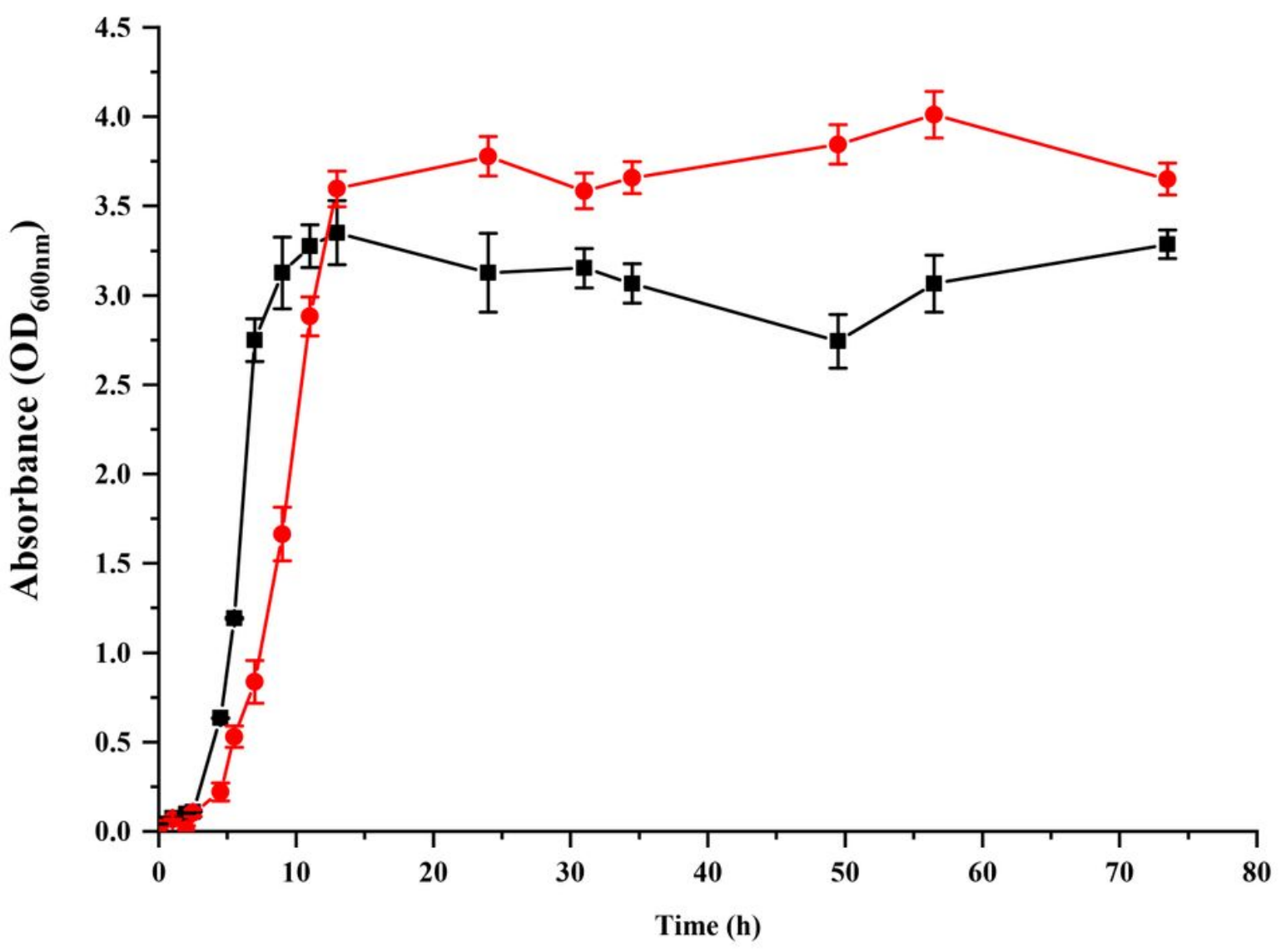


Figure 7

Growth curve of mutant and wild strains. Solid cycle, wild-type strain; Squares, Q426 $\Delta$ comX

\section{Supplementary Files}

This is a list of supplementary files associated with this preprint. Click to download.

- SupportingInformation201223.docx 